\title{
The Parisi formula completed
}

\author{
V. Janiš \\ Institute of Physics, Academy of Sciences of the Czech Republic, \\ $\mathrm{Na}$ Slovance 2, CZ-18221 Praha, Czech Republic *
}

(Dated: October 27, 2018)

\begin{abstract}
The Parisi formula for the free energy of the Sherrington-Kirkpatrick model is completed to a closed-form generating functional. We first find an integral representation for a solution of the Parisi differential equation and represent the free energy as a functional of order parameters. Then we set stationarity equations for local maxima of the free energy determining the order-parameter function on interval $[0,1]$. Finally we show without resorting to the replica trick that the solution of the stationarity equations leads to a marginally stable thermodynamic state.
\end{abstract}

PACS numbers: 64.60.Cn,75.50.Lk

Interest of physicists in spin glasses has nor been abating during last few decades. In spite of a tremendous progress in understanding of, in particular, the meanfield theory of spin glasses achieved in recent years, answers to a number of questions about physical properties of spin-glass models have not yet been found with ultimate validity. Although presently the major theoretical effort concentrates on clarifying the relevance of the mean-field solution for finite-dimensional systems, there still remain unresolved issues on the mean-field level.

The paragon mean-field theory for spin glasses is provided by the Sherrington-Kirkpatrick (SK) model introduced more than thirty years ago [1]. It took only a few years before Parisi inferred by a specific ansatz the form of a consistent solution of this model [2]. Parisi's solution, however, did not mark the end but rather the beginning of interest of theorists in spin-glass systems. There were two major reasons for further investigations of properties of the SK model and the Parisi solution. First, although most of the properties of the Parisi solution indicated that it is the exact solution of the SK model, no mathematical proof existed in that time. Second, the Parisi solution was derived via the formal replica trick and the order parameters necessary for its description were constructed from non-measurable mathematical objects. The proper physical meaning of the replicasymmetry breaking (RSB) in the spin-glass phase was initially unclear.

The principal breakthrough in the proof of the exactness of the Parisi solution was achieved only a few years ago by Guerra and Talagrand [3, 4]. They succeeded in proving rigorously that the replica-symmetry scheme of Parisi represents simultaneously both a lower as well as an upper bound on the free energy of the SK model in the thermodynamic limit. The existence of the thermodynamic limit with the self-averaging property of the free energy had already been proved earlier [5]. Unfortunately, neither the Parisi formula for the free energy nor the construction of Guerra and Talagrand do provide equations determining uniquely the thermodynamic state in the spin-glass phase. Although Talagrand con- jectures the existence of a unique macroscopic state [6], the Parisi free energy is expressed only in a loose form of a formal maximum in a large functional space of orderparameter functions. The way how to approach practically the maximum and how does the order-parameter function look like remain presently unset. Moreover, not having an explicit representation for the free energy stationary with respect to (w.r.t.) order parameters, it is not straightforward to define physical quantities such as magnetic susceptibility, entropy or specific heat.

The aim of this Letter is to extend the Parisi expression for the free energy of the SK model to an explicit closed-form functional of the order parameters. Maximizing the free energy, unspecified in the Parisi formulation, will then become a uniquely defined process of finding solutions to stationarity equations determining fully the actual form and values of all order parameters. The Parisi free energy extended in this way becomes a functional containing the entire physical information and generating all physical quantities via (functional) derivatives without referring to the replica trick and mathematical replicas. Moreover, our formulation of the mean-field free energy opens a way to systematic expansions and nonperturbative approximations to physical quantities in the low-temperature spin-glass phase.

Using the replica trick, Parisi expressed the free energy of the SK model as a functional of the order parameter function $q(x)$ for $x \in[0,1]$ generalizing the single Sherrington-Kirkpatrick order parameter $q=$ $N^{-1} \sum_{i} m_{i}^{2}$, where $m_{i}$ are local magnetizations. The free energy density in the Parisi solution is then expressed as [2]

$$
\begin{aligned}
F_{T}[q]= & -\frac{\beta^{2}}{4}\left(1+\int_{0}^{1} q(x)^{2} d x-2 q(1)\right) \\
& \quad-\int_{-\infty}^{\infty} \mathcal{D} \eta f(0, h+\eta \sqrt{q(0)}), \\
F_{P}= & \max _{q(x)} T F_{T}[q]
\end{aligned}
$$

where we used an abbreviation for a Gaussian differential $\mathcal{D} \eta \equiv \mathrm{d} \eta e^{-\eta^{2} / 2} / \sqrt{2 \pi}$. The most difficult "interacting" 
part of the above free energy $f(x, h)$ is not known explicitly. It is merely characterized by a Parisi differential equation with an initial condition

$$
\begin{aligned}
\frac{\partial f(x, h)}{\partial x} & =-\frac{1}{2} \frac{d q}{d x}\left[\frac{\partial^{2} f(x, h)}{\partial h^{2}}+x\left(\frac{\partial f(x, h)}{\partial h}\right)^{2}\right], \\
f(1, h) & =\ln [2 \cosh \beta h]
\end{aligned}
$$

the physical functional must obey. The physical solution for the free energy should then be constructed by picking up the function $q(x)$, being nondecreasing on interval $[0,1]$, so that functional $f(x, h)$ obeying Eq. (2) maximizes the free energy from Eq. (11). We, however, do not know whether the maximizing order-parameter function $q(x)$ obeys a specific equation and if yes, how does the equation look like.

We can gain some insight into the phase space of the order-parameter functions from the Guerra and Talagrand construction. It relies on the so-called discrete replica-symmetry breaking scheme. The latter can be derived straightforwardly from demanding thermodynamic homogeneity of the resulting free energy. Thermodynamic homogeneity is tested by stability of free energies with replicated spin systems w.r.t. a weak interaction between the replicated spins [7]. We replicate the original system so many times until we reach thermodynamic homogeneity, that is, independence of a further replication. In this way a hierarchical structure of free energy emerges due to successive replications of the original system. The averaged free energy density with $K$ hierarchies can then be represented as a functional of local response functions to the inter-replica interaction [7]

$$
\begin{gathered}
f^{K}\left(q, \Delta \chi_{1}, \ldots, \Delta \chi_{K} ; m_{1}, \ldots, m_{K}\right)=-\frac{1}{\beta} \ln 2 \\
+\frac{\beta}{4} \sum_{l=1}^{K} m_{l} \Delta \chi_{l}\left[2\left(q+\sum_{i=l+1}^{K} \Delta \chi_{i}\right)+\Delta \chi_{l}\right] \\
-\frac{\beta}{4}\left(1-q-\sum_{l=1}^{K} \Delta \chi_{l}\right)^{2}-\frac{1}{\beta} \int_{-\infty}^{\infty} \mathcal{D} \eta \ln Z_{K} .
\end{gathered}
$$

We used a sequence of partition functions

$$
Z_{l}=\left[\int_{-\infty}^{\infty} \mathcal{D} \lambda_{l} Z_{l-1}^{m_{l}}\right]^{1 / m_{l}},
$$

the initial condition for which reads

$Z_{0}=\cosh \left[\beta\left(h+\eta \sqrt{q}+\sum_{l=1}^{K} \lambda_{l} \sqrt{\Delta \chi_{l}}\right)\right]$. We again denoted the Gaussian differential $\mathcal{D} \lambda$ introduced in Eq. (11). The order parameter $q$ is the only one directly connected with local magnetizations. The other ones, $1>\Delta \chi_{1} \geq$ $\Delta \chi_{2} \geq \ldots \Delta \chi_{K} \geq 0$ and $1 \geq m_{1} \geq m_{2} \geq \ldots m_{k} \geq 0$ were introduced due to an induced interaction between replicated spins. All the order parameters are determined from stationarity equations locally maximizing free energy (3). The number of hierarchies $K$ is not an order parameter characterizing a saddle point of the free energy. It is fixed from stability conditions, that is, it is a number of steps needed for achieving thermodynamic homogeneity [7].

It was actually the discrete form of the replicasymmetry breaking that was used by Guerra and Talagrand to prove its exactness for the free energy of the SK model. They proved that free energy (3) becomes exact for the set of pairs $\left\{m_{1}, \Delta \chi_{1}, m_{2}, \Delta \chi_{2}, \ldots, m_{K}, \Delta \chi_{K}\right\}$ for which it is maximal. It is not specified whether the set is finite or infinite, how the parameters should be distributed on the underlying interval $[0,1]$, or whether they obey specific (stationarity) equations. The extremum may well become a supremum reached only at the boundary of the multidimensional phase space.

It is important to realize that the discrete free energy, Eq. (3), and the continuous one, Eqs. (1) and (2), are not identical. First, the former has two sets of order parameters $m_{l}$ and $\Delta \chi_{l}$ for $l=1, \ldots, K$ while the latter only one, $q(x)$ for $x \in[0,1]$. Second, the order parameters from the discrete hierarchical free energy are determined from stationarity equations unlike the Parisi free energy, where the equation for $q(x)$ is unknown. Third, the discrete free energy generally does not obey the Parisi differential equation (2). In fact, the Parisi free energy emerges from a specific limit of the discrete ones, namely when $K \rightarrow \infty, \Delta \chi_{l}=\Delta \chi / K \rightarrow d x$, and we neglect second and higher powers of $\Delta \chi_{l}$ with the fixed index $l$ 7].

Parisi's solution is defined on a subclass of measures considered by Guerra and Talagrand on which we look for a maximum (supremum). It seems that at least for the SK model, continuous of the Parisis solution measures form a complete space and the Parisi free energy determines the exact, marginally stable solution. We demonstrated it explicitly in the asymptotic region below the critical temperature of the spin-glass phase in zero magnetic field [8]. On the other hand, there are models, such as the Potts spin glass [9], where a discrete one-step RSB appears to be stable on a finite temperature interval [10].

We demonstrate in this Letter that independently of where the absolute maximum of the RSB free energy may lie, we can always construct a solution with a continuous distribution of differences $\Delta \chi_{l}$ and a single orderparameter function $m(\lambda)$ on the defining interval $[0,1]$ determined from an explicit equation for a local maximum of the free energy.

To formulate the continuous free energy and to fix 0 and 1 as ends of the underlying interval on which the order-parameter function is defined we introduce two physical order parameters $q$ and $X$. The former corresponds to $q(0)$ and the latter to $q(1)-q(0)$ in the Parisi solution. We do not use the sequence $1 \geq m_{1}, \geq \ldots m_{K} \geq 0$ to set the interval on which the order-parameter function is defined as in Eq. (1). We find it more convenient to reverse the choice and use $\Delta \chi_{l}=X d \lambda$ as the fundamental 
infinitesimal differential. Neglecting all higher than linear powers of $\Delta \chi_{l}$, unless accompanied by a compensating summation over the labeling indices, free energy (3) reduces to

$$
\begin{gathered}
f(q, X ; m(\lambda))=-\frac{\beta}{4}(1-q-X)^{2}-\frac{1}{\beta} \ln 2 \\
+\frac{\beta X}{2} \int_{0}^{1} d \lambda m(\lambda)[q+X(1-\lambda)]-\frac{1}{\beta}\langle g(1, h+\eta \sqrt{q})\rangle_{\eta}
\end{gathered}
$$

where $\langle X(\eta)\rangle_{\eta}=\int_{-\infty}^{\infty} \mathcal{D} \eta X(\eta)$. The principal achievement of this Letter is an explicit integral representation of the interacting free energy $g(1, h)$. Dropping all terms of order $O\left(d \lambda^{2}\right)$ in the discrete hierarchy of partition sums $Z_{l}$ in the continuous limit $K \rightarrow \infty$ with $\Delta \chi_{l}=\Delta \chi / K=X d \lambda$ we finally obtain

$$
\begin{aligned}
& g(1, h)= \mathbb{E}_{0}(X, h ; 1,0) \circ g_{0}(h) \\
& \equiv \mathbb{T}_{\lambda} \exp \left\{\frac { X } { 2 } \int _ { 0 } ^ { 1 } d \lambda \left[\partial_{\bar{h}}^{2}\right.\right. \\
&\left.\left.\quad+m(\lambda) g^{\prime}(\lambda ; h+\bar{h}) \partial_{\bar{h}}\right]\right\}\left.g_{0}(h+\bar{h})\right|_{\bar{h}=0},
\end{aligned}
$$

where we used prime to denote a derivative w.r.t. the magnetic field $h, g^{\prime}(\lambda, h) \equiv \partial_{h} g(\lambda, h)$. To reach a closed form for the continuous free energy we introduced a "time-ordering" operator $\mathbb{T}_{\lambda}$ ordering products of $\lambda$ dependent non-commuting operators from left to right in a $\lambda$-decreasing succession. The time-ordered exponential is then defined as

$$
\begin{aligned}
& \mathbb{T}_{\lambda} \exp \left\{\int_{0}^{1} d \lambda \widehat{O}(\lambda)\right\} \equiv 1 \\
& +\sum_{n=1}^{\infty} \int_{0}^{1} d \lambda_{1} \int_{0}^{\lambda_{1}} d \lambda_{2} \ldots \int_{0}^{\lambda_{n-1}} d \lambda_{n} \widehat{O}\left(\lambda_{1}\right) \ldots \widehat{O}\left(\lambda_{n}\right)
\end{aligned}
$$

Time-ordering operators are a standard tool in representing quantum many-body perturbation expansions. The initial condition for the $\lambda$-evolution in Eq. (5) is the local free energy $g_{0}(h)=\ln [\cosh \beta h]$. Unlike the Parisi construction we develop the solution on the defining interval from zero to one.

It is straightforward to verify that the interacting part of the free energy $g(\lambda, h)$ obeys a Parisi-like equation

$$
\frac{\partial g(\lambda, h)}{\partial \lambda}=\frac{X}{2}\left[\frac{\partial^{2} g(\lambda, h)}{\partial h^{2}}+m(\lambda)\left(\frac{\partial g(\lambda, h)}{\partial h}\right)^{2}\right] \text {. }
$$

The opposite overall sign of the r.h.s. of this equation compared with Eq. (2) is connected with the reverted evolution of the initial condition used here.

Evolution operator $\mathbb{E}_{0}(X ; \mu, \nu)$ contains only polynomials in powers of derivatives w.r.t. an auxiliary magnetic field replacing the Gaussian integration over auxil- iary random fields $\lambda_{l}$ in Eq. (3). It is, however, a nonlinear operator that is why we must find analogous integral representations for functions $g^{\prime}(\lambda, h)$ and $g^{\prime \prime}(\lambda, h)$ appearing in Eq. (6).

From the definition of the evolution operator $\mathbb{E}_{0}$ we obtain

$$
\begin{aligned}
& \frac{\partial g(\lambda, h)}{\partial h}=\mathbb{E}_{0}(X, h ; \lambda, 0) \circ g_{0}^{\prime}(h) \\
+ & \frac{X}{2} \int_{0}^{\lambda} d \nu m(\nu) \mathbb{E}_{0}(X, h ; \lambda, \nu) \circ\left[g^{\prime}(\nu, h) \partial_{h} g^{\prime}(\nu, h)\right] .
\end{aligned}
$$

A solution to this integral equation can again be represented via an evolution operator and the $\mathbb{T}$-ordered exponential

$$
\begin{aligned}
& g^{\prime}(\nu, h)= \mathbb{E}(X, h ; \nu, 0) \circ g_{0}^{\prime}(h) \\
& \equiv \mathbb{T}_{\lambda} \exp \left\{X \int _ { 0 } ^ { \nu } d \lambda \left[\frac{1}{2} \partial_{\bar{h}}^{2}\right.\right. \\
&\left.\left.\quad+m(\lambda) g^{\prime}(\lambda ; h+\bar{h}) \partial_{\bar{h}}\right]\right\}\left.g_{0}^{\prime}(h+\bar{h})\right|_{\bar{h}=0}
\end{aligned}
$$

Analogously we derive an evolution operator for the second derivative of the free energy

$$
\begin{aligned}
& g^{\prime \prime}(\nu, h)= \mathbb{E}_{2}(X, h ; 1,0) \circ g_{0}^{\prime \prime}(h) \\
& \equiv \mathbb{T}_{\lambda} \exp \left\{X \int _ { 0 } ^ { \nu } d \lambda \left[\frac{1}{2} \partial_{\bar{h}}^{2}\right.\right. \\
&\left.\left.\quad+m(\lambda) \partial_{\bar{h}} g^{\prime}(\lambda ; h+\bar{h})\right]\right\}\left.g_{0}^{\prime \prime}(h+\bar{h})\right|_{\bar{h}=0} .
\end{aligned}
$$

Having an explicit representation for the free energy we can derive stationarity equations for its local extrema. Free energy $f(q, X ; m(\lambda))$ is a function of static parameters $q$ and $X$ and a nonlinear functional of the dynamical order-parameter function $m(\lambda)$. Vanishing of the free energy w.r.t. infinitesimal variations the static parameters leads to the following equations

$$
\begin{aligned}
q= & \frac{1}{\beta^{2}}\left\langle g^{\prime}\left(1, h_{\eta}\right)^{2}\right\rangle_{\eta}, \\
X=\frac{1}{\beta^{2}} & {\left[\left\langle\mathbb{E}\left(X, h_{\eta} ; 1,0\right) \circ g_{0}^{\prime}\left(h_{\eta}\right)^{2}\right\rangle_{\eta}\right.} \\
& \left.\quad-\left\langle g^{\prime}\left(1, h_{\eta}\right)^{2}\right\rangle_{\eta}\right] .
\end{aligned}
$$

We denoted $h_{\eta} \equiv h+\eta \sqrt{q}$. Vanishing of the free energy w.r.t. infinitesimal variations of function $m(\lambda)$ leads to a functional equation

$$
\begin{aligned}
\lambda= & \frac{1}{\beta^{2} X}\left[\left\langle\mathbb{E}\left(X, h_{\eta} ; 1,0\right) \circ g_{0}^{\prime}\left(h_{\eta}\right)^{2}\right\rangle_{\eta}\right. \\
& \left.-\left\langle\mathbb{E}\left(X, h_{\eta} ; 1, \lambda\right) \circ g^{\prime}\left(\lambda, h_{\eta}\right)^{2}\right\rangle_{\eta}\right]
\end{aligned}
$$


valid for any $\lambda \in[0,1]$. Notice that Eq. (10c) for $\lambda=0$ is trivial and for $\lambda=1$ coincides with Eq. (10b). Hence, only equations for $0<\lambda<1$ serve for the determination of $m$ as a function of the evolution parameter $\lambda$.

Free energy (4) complemented with stationarity equations (10) defines a thermodynamic state of the SK model for all input parameters. It is thermodynamically consistent so that physical values of all internal parameters specifying the thermodynamic state are determined selfconsistently from stationarity equations and the standard thermodynamic relations hold. For instance, magnetic susceptibility reads

$$
\chi_{T}=\frac{1}{\beta}\left\langle g^{\prime \prime}(1, h+\eta \sqrt{q})\right\rangle_{\eta} .
$$

We do not have an integral representation such as in the Parisi formulation [2], since we do not use $q(x)$ as the order-parameter function but rather $m(\lambda)$. Nevertheless, we have an alternative implicit representation of $g^{\prime \prime}(\lambda, h)$ in Eq. (9). It is, however, important that we do not need to resort to the replica trick to define and calculate physical quantities in the thermodynamic state described by free energy (4).

One of the attractive features of the presented extension of the Parisi free energy is a possibility to verify stability of the solution of equations (10). To this purpose we utilize the fact that Eq. (10c) holds for all $\lambda \in[0,1]$. Then also a total derivative of both sides w.r.t. $\lambda$ must be equal. Employing properties of the evolution operator $\mathbb{E}$ we find

$$
\begin{aligned}
& \frac{d}{d \lambda} \mathbb{E}(X, h ; 1, \lambda) \circ g^{\prime}(\lambda, h)^{2} \\
&=-X \mathbb{E}(X, h ; 1, \lambda) \circ g^{\prime \prime}(\lambda, h)^{2} .
\end{aligned}
$$

Using this result in Eq. (10c) we obtain a generalization of the de Almeida-Thouless condition [11] for marginal thermodynamic stability

$$
\beta^{2}=\left\langle\mathbb{E}\left(X, h_{\eta} ; 1, \lambda\right) \circ g^{\prime \prime}\left(\lambda, h_{\eta}\right)^{2}\right\rangle_{\eta} .
$$

It is actually a functional equation for $\lambda \in[0,1]$. With some effort we can verify that Eq. (13) is a continuous limit of stability conditions derived in [7, 12]. We see that the Parisi solution of the SK model satisfying Eqs. (10) is marginally stable with no negative eigenvalues of the nonlocal susceptibility or the spin-glass susceptibility.

Free energy (44) with the interacting part from Eq. (5) is defined implicitly, since the evolution operator $\mathbb{E}_{0}$ contains the solution $g^{\prime}(\lambda, h)$. This cannot be avoided, since the Parisi differential equation (6) is nonlinear. We can, nevertheless use the implicit integral representation of the evolution operator to an approximate computation of the free energy and other physical quantities. The most straightforward way is to expand the $\mathbb{T}$-ordered exponential in powers of the exponent. This practically corresponds to a power-series expansion of the orderparameter function $m(\lambda)$. Such an approach becomes asymptotically exact near the de Almeida-Thouless instability line. We hence can analyze the critical behavior without the necessity to come over to truncated a model. If one finds an effective way how to systematically generates higher-order terms of such a power expansion, a rather accurate approximation in the entire spin-glass phase can be accomplished. Another method for resolving the evolution operator is to approximate the orderparameter function with piece-wise constant functions. In this way we approximate the continuous scheme by a discrete one, resembling the discrete RSB from Eq. (3).

To conclude, we completed the Parisi formula for the free energy of the SK model so that it acquires the standard form demanded by the fundamental principles of statistical mechanics. The derived free energy is a function of two numerical order parameters $q$ and $X$ and a functional of an order-parameter function $m(\lambda)$ defined on interval $[0,1]$. The physical values of these order parameters are determined from stationarity equations for local extrema (maxima) of the free energy. The free energy thus becomes a well defined generating functional from which all physical quantities can be derived via standard thermodynamic methods. There is no need to resort to the replica trick and a representation via mathematical replicas to identify measurable quantities. The integral representation of the solution of the Parisi differential equation demonstrates that the thermodynamic state of the SK model is marginally stable and allows for explicit systematic and non-perturbative approximations of the thermodynamics of mean-field spin-glass models.

Research on this problem was carried out within a project AVOZ10100520 of the Academy of Sciences of the Czech Republic.

* Electronic address: janis@fzu.cz

[1] D. Sherrington and S. Kirkpatrick, Phys. Rev. Lett. 35, 1972 (1975).

[2] G. Parisi, J. Phys. A 13, L115, 1101 (1980).

[3] F. Guerra, Commun. Math. Phys. 233, 1 (2003).

[4] M. Talagrand, Ann. Math. 163, 221 (2006).

[5] F. Guerra and F. L. Toninelli, Commun. Math. Phys. 230, 71 (2002).

[6] M. Talagrand, C. R. Math. Acad. Sci. Paris 337, 625 (2003).

[7] V. Janiš, Phys. Rev. B 71, 214403 (2005).

[8] V. Janiš and A. Klíč, Phys. Rev. B 74, 054410 (2006).

[9] D. Elderfield and D. Sherrington, J. Phys. C16, L497 (1983).

[10] D. J. Gross, I. Kanter, and H. Sompolinsky, Phys. Rev. Lett. 55, 304 (1985).

[11] J. R. L. de Almeida and D. J. Thouless, J. Phys. A 11, 983 (1978).

[12] V. Janiš, Phys. Rev. B 74, 054207 (2006). 